\title{
LPS's Criterion for Incompressible Nematic Liquid Crystal Flows
}

\author{
Qing Chen \\ Department of Mathematics and Physics \\ Xiamen University of Technology, Xiamen 361024, China \\ Zhong Tan \\ School of Mathematical Sciences, Xiamen University, Fujian 361005, China \\ Guochun Wu* \\ School of Mathematical Sciences, Xiamen University, Fujian 361005, China
}

\begin{abstract}
In this paper we derive LPS's criterion for the breakdown of classical solutions to the incompressible nematic liquid crystal flow, a simplified version of Ericksen-Leslie system modeling the hydrodynamic evolution of nematic liquid crystals in $\mathbb{R}^{3}$. We show that if $0<T<+\infty$ is the maximal time interval for the unique smooth solution $u \in C^{\infty}\left([0, T), \mathbb{R}^{3}\right)$, then $|u|+|\nabla d| \notin L^{q}\left([0, T], L^{p}\left(\mathbb{R}^{3}\right)\right)$, where $p$ and $q$ safisfy the Ladyzhenskaya-Prodi-Serrin's condition: $\frac{3}{p}+\frac{2}{q}=1$ and $p \in(3,+\infty]$.
\end{abstract}

Keywords Incompressible nematic liquid crystal flow; Ladyzhenskaya-ProdiSerrin's criterion.

MSC2010 35Q35; 76D03.

\section{Introduction}

We consider the following hydrodynamic system modeling the flow of liquid crystal materials in dimension three (see $[2,3,10,12]$ and references therein):

$$
\begin{gathered}
u_{t}+u \cdot \nabla u-\nu \triangle u+\nabla P=-\Delta d \cdot \nabla d, \\
\partial_{t} d+u \cdot \nabla d=\Delta d+|\nabla d|^{2} d, \\
\nabla \cdot u=0 \quad|d|=1
\end{gathered}
$$

*Corresponding author. Email address: guochunwu@126com. 
for $(t, x) \in[0,+\infty) \times \mathbb{R}^{3}$. Here $u: \mathbb{R}^{3} \rightarrow \mathbb{R}^{3}$ represents the velocity field of the incompressible viscous fluid, $\nu>0$ is the kinematic viscosity, $P: \mathbb{R}^{3} \rightarrow \mathbb{R}$ represents the pressure function, and $d: \mathbb{R}^{3} \rightarrow \mathbb{S}^{2}$ represents the macroscopic average of the nematic liquid crystal orientation field. We are interested in the Cauchy problem (1.1) with the initial value

$$
(u(0, x), d(0, x))=\left(u_{0}(x), d_{0}(x)\right)
$$

satisfying the following compatibility condition:

$$
\nabla \cdot u_{0}(x)=0, \quad\left|d_{0}(x)\right|=1, \quad \lim _{|x| \rightarrow \infty} d_{0}(x)=a \in \mathbb{S}^{2},
$$

where $a$ is a given unit vector.

The above system is a simplified version of the Ericksen-Leslie model, which reduces to the Ossen-Frank model in the static case, for the hydrodynamics of nematic liquid crystals developed during the period of 1958 through 1968 [2,3,10]. It is a macroscopic continuum description of the time evolution of the materials under the influence of both the flow field $u(x, t)$, the macroscopic description of the microscopic orientation configurations $d(x, t)$ of rod-like liquid crystals. Roughly speaking, the system (1.1) is a coupling between the non-homogeneous Navier-Stokes equation and the transported flow harmonic maps. Due to the physical importance and mathematical challenges, the study on nematic liquid crystals has attracted many physicists and mathematicians. The mathematical analysis of the liquid crystal flows was initiated by Lin [11], Lin and Liu in $[12,13]$. For any bounded smooth domain in $\mathbb{R}^{2}$, Lin, Lin and Wang [14] have proved the global existence of Leray-Hopf type weak solutions to system (1.1) which are smooth everywhere except on finitely many time slices (see [5] for the whole space). The uniqueness of weak solutions in two dimension was studied by [15,20]. Recently, Hong and Xin [6] studied the global existence for general Ericksen-Leslie system in dimension two. However, the global existence of weak solutions to the incompressible nematic liquid crystal flow equation (1.1) in three dimension with large initial data is still an outstanding open question.

In this paper, we are interested in an optimal characterization on the maximal interval $T$ that is scaling invariant. So let us first introduce the following definition:

Definition 1.1. For $1 \leq p, q \leq \infty$, we say a function $f=f(t, x):[0, T] \times \mathbb{R}^{3} \rightarrow \mathbb{R}$ is in $L^{q}\left([0, T], L^{p}\left(\mathbb{R}^{3}\right)\right)$, if

$$
\begin{aligned}
\|f\|_{L^{q}\left([0, T], L^{p}\left(\mathbb{R}^{3}\right)\right)} & =\left(\int_{0}^{T}\|f(t, \cdot)\|_{L^{p}\left(\mathbb{R}^{3}\right)}^{q} d t\right)^{\frac{1}{q}}, \quad 1 \leq q<\infty \\
& =e s s \sup _{t \in[0, T]}\|f(t, \cdot)\|_{L^{p}\left(\mathbb{R}^{3}\right)}, \quad q=\infty
\end{aligned}
$$

is finite. If $p=q$, then we simply write $\|f\|_{L^{p}\left([0, T], \mathbb{R}^{3}\right)}$ for $\|f\|_{L^{p}\left([0, T], L^{p}\left(\mathbb{R}^{3}\right)\right)}$.

We will consider the short time classical solution to (1.1) and address the LadyzhenskayaProdi-Serrin's criterion that characterizes the first time finite singular time. The 
local well-posedness of the Cauchy problem of system (1.1) is rather standard (see $[5,7,14])$. More precisely, if the initial velocity $u_{0} \in H^{s}\left(\mathbb{R}^{3}, \mathbb{R}^{3}\right)$ with $\nabla \cdot u_{0}=0$ and $d_{0}-a \in H^{s+1}\left(\mathbb{R}^{2}, \mathbb{S}^{2}\right)$ such that system (1.1) has a unique, classical solution $(u, d)$ in $\left[0, T_{0}\right) \times \mathbb{R}^{3}$ satisfying

$$
\begin{aligned}
& u \in C\left([0, T), H^{s}\left(\mathbb{R}^{3}\right)\right) \cap C^{1}\left([0, T), H^{s-2}\left(\mathbb{R}^{3}\right)\right) \text { and } \\
& d-a \in C\left([0, T), H^{s+1}\left(\mathbb{R}^{3}, \mathbb{S}^{2}\right)\right) \cap C^{1}\left([0, T), H^{s-1}\left(\mathbb{R}^{3}, \mathbb{S}^{2}\right)\right),
\end{aligned}
$$

for any $0<T<T_{0}$. At present, there is no global-in-time existence theory for classical solutions to system (1.1). Thus if we assume $T_{*}>0$ is the maximum value such that (1.4) holds with $T_{0}=T_{*}$, we would like to characterize such a $T_{*}$. Motivated by the famous work [1], Huang and Wang [7] have obtained a $B K M$ type blow-up criterion (see also [16]). However, the techniques involved in this paper are much different from [7], which we believe that the result may have its own interest.

When $d$ is a constant vector field, the system (1.1) becomes an incompressible Navier-Stokes equation. Recall that the scaling invariant space $L^{q}\left([0, T], L^{p}\left(\mathbb{R}^{3}\right)\right)$, with $(p, q)$ satisfying

$$
\frac{3}{p}+\frac{2}{q}=1
$$

which has played an important role in the regularity issue of Navier-Stokes equation. Leray [9] first established the existence of a global weak solution for Navier-Stokes equation, now called Leray-Hopf weak solution, that satisfies an energy inequality:

$$
\frac{1}{2}\|u(t)\|_{L^{2}\left(\mathbb{R}^{3}\right)}^{2}+\int_{0}^{t} \int_{\mathbb{R}^{3}}|\nabla u(t, x)|^{2} d x d s \leq \frac{1}{2}\left\|u_{0}\right\|_{L^{2}\left(\mathbb{R}^{3}\right)}^{2} .
$$

Although the regularity issue for Leray-Hopf weak solutions of Navier-Stokes equation remains open, it is well-known that both uniqueness and smoothness for the class of weak solutions of Navier-Stokes equation, in which $u \in L^{q}\left([0, T], L^{p}\left(\mathbb{R}^{3}\right)\right)$ for some $p \in(3,+\infty]$ and $q \in[2,+\infty)$ satisfying Ladyzhenskaya-Prodi-Serrin's condition (1.5) have been established through works by Prodi [18], Serrin [19], and Ladyzhenskaya [8] in 1960s. On the other hand, for the end point case $p=3, q=+\infty$, only until very recently Escauriaza et al. [4] have finally proved the smoothness for weak solution $u \in L^{\infty}\left([0, T], L^{3}\left(\mathbb{R}^{3}\right)\right)$ of incompressible Navier-Stokes equation, $0<T \leq+\infty$.

Motivated by these results for the Navier-Stokes equation, we are going to use scaling considerations for system (1.1) to guess which spaces may be critical. We observe that system (1.1) is invariant by the following transformation:

$$
\hat{u}=l u\left(l^{2} t, l x\right), \quad \hat{P}=l^{2} P\left(l^{2} t, l x\right), \quad \hat{d}=d\left(l^{2} t, l x\right) .
$$

Thus $L^{q}\left([0, T], L^{p}\left(\mathbb{R}^{3}\right)\right)$ is a critical space for $(u, \nabla d)$ if $(p, q)$ satisfies the LadyzhenskayaProdi-Serrin's condition (1.5).

Our main results are formulated as the following theorem:

Theorem 1.1. For $u_{0} \in H^{s}\left(\mathbb{R}^{3}\right)$ with $\nabla \cdot u_{0}=0$ and $d_{0}-a \in H^{s+1}\left(\mathbb{R}^{3}\right)$ with 
$\left|d_{0}\right|=1$ for $s \geq 3$. Suppose that $(u, d)$ is a smooth solution to the system (1.1)-(1.2), then for given $T>0,(u, d)$ is smooth up to time $T$ provided that

$$
\|u\|_{L^{q}\left([0, T], L^{p}\left(\mathbb{R}^{3}\right)\right)}+\|\nabla d\|_{L^{q}\left([0, T], L^{p}\left(\mathbb{R}^{3}\right)\right)}<+\infty,
$$

where $(p, q)$ satisfies the Ladyzhenskaya-Prodi-Serrin's condition $(1.5)$ and $p \in(3,+\infty]$.

Notations. We denote by $L^{p}, W^{m, p}$ the usual Lebesgue and Sobolev spaces on $\mathbb{R}^{3}$ and $H^{m}=W^{m, 2}$, with norms $\|\cdot\|_{L^{p}},\|\cdot\|_{W^{m, p}}$ and $\|\cdot\|_{H^{m}}$ respectively. For the sake of conciseness, we do not distinguish functional space when scalar-valued or vector-valued functions are involved. We denote $\nabla=\partial_{x}=\left(\partial_{1}, \partial_{2}, \partial_{3}\right)$, where $\partial_{i}=\partial_{x_{i}}, \nabla_{i}=\partial_{i}$ and put $\partial_{x}^{l} f=\nabla^{l} f=\nabla\left(\nabla^{l-1} f\right)$. We assume $C$ be a positive generic constant throughout this paper that may vary at different places and the integration domain $\mathbb{R}^{3}$ will be always omitted without any ambiguity. Finally, $\langle\cdot, \cdot\rangle$ denotes the inner-product in $L^{2}\left(\mathbb{R}^{3}\right)$.

Remark 1.1. It is standard that the condition (1.3) is preserved by the flow. In fact, first notice that the divergence free of the velocity field $u$ can be justified by the initial assumption that $\nabla \cdot u_{0}=0$. Indeed, this can be easily and formally observed by take $\nabla \cdot$ to the momentum equation. Moreover, applying the maximum principle to the equation for $|d|^{2}$, one also can easily see that $|d|=1$ under the initial assumption that $\left|d_{0}\right|=1$.

\section{Proof of Theorem 1.1}

We prove our theorem in this section. Without loss of generality, we assume $\nu=1$. The first bright idea to reduce many complicated computations lies in that we just need to do the lowest order and highest order energy estimates for the solutions. This is motivated by the following observation:

$$
\|f\|_{H^{k}}^{2} \leq C\left\|\left(f, \nabla^{k} f\right)\right\|_{L^{2}}^{2}, \quad \forall f \in H^{k} .
$$

This inequality (2.1) can be easily proved by combing Young's inequality and GagliardoNirenberg's inequality.

$$
\left\|\nabla^{i} f\right\|_{L^{p}} \leq C(p)\|f\|_{L^{q}}^{\alpha}\left\|\nabla^{k} f\right\|_{L^{r}}^{1-\alpha}, \quad \forall f \in H^{k}
$$

where $\frac{1}{p}-\frac{i}{3}=\frac{1}{q} \alpha+\left(\frac{1}{r}-\frac{k}{3}\right)(1-\alpha)$ with $i \leq k$.

Now we are in a position to prove our Theorem 1.1.

Proof of Theorem 1.1 First of all, we note that if $p=+\infty$, Theorem 1.1 has been proved in [16], thus let us concentrate on $p \in(3,+\infty)$. Now for classical solutions to (1.1)-(1.2), one has the following basic energy law:

$$
\begin{array}{cc}
\|u(t, \cdot)\|_{L^{2}}^{2}+\|\nabla d(t, \cdot)\|_{L^{2}}^{2} & +\int_{0}^{t}\left(\|\nabla u(s, \cdot)\|_{L^{2}}^{2}+\left\|\triangle d(s, \cdot)+|\nabla d|^{2} d(s, \cdot)\right\|_{L^{2}}^{2}\right) d s \\
= & \left\|u_{0}\right\|_{L^{2}}^{2}+\left\|\nabla d_{0}\right\|_{L^{2}}^{2}, \quad \forall t>0 .
\end{array}
$$


Let's concentrate on the case $s=3$. For each multi-index $\alpha$ with $|\alpha| \leq 3$, by applying $\partial_{x}^{\alpha}$ to (1.1a) and $\partial_{x}^{\alpha+1}$ to (1.1b), multiplying them by $\partial_{x}^{\alpha} u, \partial_{x}^{\alpha+1} d$ respectively and then integrating them over $\mathbb{R}^{3}$, we have

$$
\begin{aligned}
& \frac{1}{2} \frac{d}{d t}\left\|\partial_{x}^{\alpha}(u, \nabla d)\right\|_{L^{2}}^{2}+\left\|\partial_{x}^{\alpha}(\nabla u, \triangle d)\right\|_{L^{2}}^{2} \\
= & -\left\langle\partial_{x}^{\alpha}(u \cdot \nabla u), \partial_{x}^{\alpha} u\right\rangle-\left\langle\partial_{x}^{\alpha}(\triangle d \cdot \nabla d), \partial_{x}^{\alpha} u\right\rangle \\
& -\left\langle\partial_{x}^{\alpha+1}(u \cdot \nabla d), \partial_{x}^{\alpha+1} d\right\rangle+\left\langle\partial_{x}^{\alpha+1}\left(|\nabla d|^{2} d\right), \partial_{x}^{\alpha+1} d\right\rangle \\
= & \sum_{i=1}^{4} I_{|\alpha|, i} .
\end{aligned}
$$

where $I_{|\alpha|, i}$ are the corresponding terms in the above equation which will be estimated as follows. Now for $|\alpha|=1$ in (2.4), integrating by parts and using the divergence free condition $\nabla \cdot u=0$ and $(2.2)$, we arrive at

$$
\begin{aligned}
\left|I_{1,1}\right| & =\left|\left\langle\partial_{x}^{1}(u \cdot \nabla u), \partial_{x}^{1} u\right\rangle\right|=\left|\left\langle\partial_{x}^{1} u \cdot \nabla u, \partial_{x}^{1} u\right\rangle\right| \leq C \int_{\mathbb{R}^{3}}|\nabla u|^{3} d x \\
& \leq C\|u\|_{L^{p}}^{\frac{3 p}{6+p}}\left\|\nabla^{2} u\right\|_{L^{2}}^{\frac{18}{6+p}} \leq C\|u\|_{L^{p}}^{\frac{2 p p}{p-3}}\|u\|_{L^{p}}^{p-3}+\frac{1}{16}\left\|\nabla^{2} u\right\|_{L^{2}}^{2} .
\end{aligned}
$$

Combining Cauchy's inequality, Sobolev's inequality and the fact $|\nabla d|^{2}=-d \cdot \Delta d$ (since $|d|=1$ ) gives

$$
\begin{aligned}
\left|I_{1,2}\right| & =\left|\left\langle\partial_{x}^{1}(\triangle d \cdot \nabla d), \partial_{x}^{1} u\right\rangle\right|=|\langle\triangle d \cdot \nabla d, \Delta u\rangle| \\
& \leq C\|\triangle d\|_{L^{3}}^{3}+\frac{1}{16}\left\|\nabla^{2} u\right\|_{L^{2}}^{2} \\
& \leq C\|\nabla d\|_{L^{\frac{3 p}{p}}}^{6+p}\left\|\nabla^{3} d\right\|_{L^{2}}^{\frac{18}{6+p}}+\frac{1}{16}\left\|\nabla^{2} u\right\|_{L^{2}}^{2} \\
& \leq C\|\nabla d\|_{L^{p}}^{\frac{2 p}{p-3}}\|\nabla d\|_{L^{p}}^{p-3}+\frac{1}{16}\left(\left\|\nabla^{3} d\right\|_{L^{2}}^{2}+\left\|\nabla^{2} u\right\|_{L^{2}}^{2}\right) .
\end{aligned}
$$

Similarly,

$$
\begin{aligned}
\left|I_{1,3}\right|= & \left|\left\langle\partial_{x}^{2} u \cdot \nabla d+\partial_{x} u \cdot \nabla \partial_{x} d, \partial_{x}^{2} d\right\rangle\right| \\
\leq & C\|\triangle d\|_{L^{3}}^{3}+\frac{1}{32}\left\|\nabla^{2} u\right\|_{L^{2}}^{2}+C\|\nabla u\|_{L^{3}}^{3} \\
\leq & C\left(\|u\|_{L^{p}}^{\frac{p p}{p-3}}\|u\|_{L^{p}}^{\frac{p p}{p-3}}+\|\nabla d\|_{L^{p}}^{\frac{2 p}{p-3}}\|\nabla d\|_{L^{p}}^{\frac{p}{p-3}}\right) \\
& +\frac{1}{16}\left(\left\|\nabla^{3} d\right\|_{L^{2}}^{2}+\left\|\nabla^{2} u\right\|_{L^{2}}^{2}\right) \\
\left|I_{1,4}\right|= & \left|\left\langle\partial_{x}^{1}\left(|\nabla d|^{2} d\right), \partial_{x} \triangle d\right\rangle\right| \\
\leq & C\left(\|\nabla d\|_{L^{6}}^{6}+\left\|\nabla^{2} d\right\|_{L^{3}}^{3}\right)+\frac{1}{32}\|\nabla \triangle d\|_{L^{2}}^{2} \\
\leq & C\left\|\nabla^{2} d\right\|_{L^{3}}^{3}+\frac{1}{32}\|\nabla \triangle d\|_{L^{2}}^{2} \\
\leq & C\|\nabla d\|_{L^{p}}^{\frac{2 p}{p-3}}\|\nabla d\|_{L^{p}}^{\frac{p}{p-3}}+\frac{1}{16}\left\|\nabla^{3} d\right\|_{L^{2}}^{2} .
\end{aligned}
$$

Taking the above estimates (2.5)-(2.8) in (2.4) for $|\alpha|=1$, we arrive at

$$
\begin{aligned}
& \frac{d}{d t}\left\|\left(\nabla u, \nabla^{2} d\right)\right\|_{L^{2}}^{2}+C\left\|\left(\nabla^{2} u, \nabla^{3} d\right)\right\|_{L^{2}}^{2} \\
\leq & C\left(\|u\|_{L^{p}}^{\frac{2 p}{p-3}}\|u\|_{L^{p}}^{p-3}+\|\nabla d\|_{L^{p}}^{\frac{2 p}{p-3}}\|\nabla d\|_{L^{p}}^{p-3}\right) .
\end{aligned}
$$

Next we derive an estimate for $\|u\|_{L^{p}}^{p}$ and $\|\nabla d\|_{L^{p}}^{p}$. First of all, we multiply (1.1a) by $|u|^{p-2} u$ and integrate over $\mathbb{R}^{3}$ to obtain that

$$
\begin{aligned}
& \frac{1}{p} \frac{d}{d t}\|u\|_{L^{p}}^{p}+\int_{\mathbb{R}^{3}}|u|^{p-2}|\nabla u|^{2} d x+\frac{1}{4}(p-2) \int_{\mathbb{R}^{3}}|u|^{p-4}\left|\nabla\left(|u|^{2}\right)\right|^{2} d x \\
= & -\left\langle\nabla P,|u|^{p-2} u\right\rangle-\left\langle\triangle d \nabla d,|u|^{p-2} u\right\rangle .
\end{aligned}
$$


Observe that

$$
\triangle d \cdot \nabla d=\nabla \cdot\left(\nabla d \odot \nabla d-\frac{1}{2}|\nabla d|^{2} I\right),
$$

where $\nabla d \odot \nabla d$ denotes the $3 \times 3$ matrix whose $(i, j)$-the entry is given by $\partial_{i} d \cdot \partial_{j} d$ for $1 \leq i, j \leq 3$. And taking div to (1.1a), we arrive at

$$
\triangle P=-\operatorname{divdiv}\left(u \otimes u+\nabla d \odot \nabla d-\frac{1}{2}|\nabla d|^{2} I\right) .
$$

An application of the $L^{p}$-estimate of elliptic systems to the above equation, there exists $\bar{P}(t)$ such that

$$
\begin{aligned}
& \int_{\mathbb{R}^{3}}|P-\bar{P}(t)|^{\frac{p+2}{2}} d x \\
\leq & C \int_{\mathbb{R}^{3}}|u|^{p+2}+|\nabla d|^{p+2} d x \\
\leq & C\left\{\left(\int_{\mathbb{R}^{3}}|u|^{p} d x\right)^{\frac{p-1}{p}}\left(\int_{\mathbb{R}^{3}}|u|^{3 p} d x\right)^{\frac{1}{p}}\right. \\
& \left.+\left(\int_{\mathbb{R}^{3}}|\nabla d|^{p} d x\right)^{\frac{p-1}{p}}\left(\int_{\mathbb{R}^{3}}|\nabla d|^{3 p} d x\right)^{\frac{1}{p}}\right\} \\
\leq \quad & C\left\{\left(\int_{\mathbb{R}^{3}}|u|^{p} d x\right)^{\frac{p-1}{p}}\left(\int_{\mathbb{R}^{3}}|u|^{p-2}|\nabla u|^{2} d x\right)^{\frac{3}{p}}\right. \\
& \left.+\left(\int_{\mathbb{R}^{3}}|\nabla d|^{p} d x\right)^{\frac{p-1}{p}}\left(\int_{\mathbb{R}^{3}}|\nabla d|^{p-2}\left|\nabla^{2} d\right|^{2} d x\right)^{\frac{3}{p}}\right\} \\
\leq \quad & C\left\{\left(\int_{\mathbb{R}^{3}}|u|^{p} d x\right)^{\frac{p-1}{p-3}}+\left(\int_{\mathbb{R}^{3}}|\nabla d|^{p} d x\right)^{\frac{p-1}{p-3}}\right\} \\
& +\frac{1}{32}\left(\int_{\mathbb{R}^{3}}|u|^{p-2}|\nabla u|^{2} d x+\int_{\mathbb{R}^{3}}|\nabla d|^{p-2}\left|\nabla^{2} d\right|^{2} d x\right) \\
\leq & C\left\{\|u\|_{L^{p}}^{p-3}\|u\|_{L^{p}}^{p}+\|\nabla d\|_{L^{p}}^{\frac{2 p}{p-3}}|| \nabla d \|_{L^{p}}^{p}\right\} \\
& +\frac{1}{32}\left(\int_{\mathbb{R}^{3}}|u|^{p-2}|\nabla u|^{2} d x+\int_{\mathbb{R}^{3}}|\nabla d|^{p-2}\left|\nabla^{2} d\right|^{2} d x\right) .
\end{aligned}
$$

Thus we have

$$
\begin{aligned}
& \left|\left\langle\nabla P,|u|^{p-2} u\right\rangle\right|=\left|\left\langle P-\bar{P}(t), \operatorname{div}\left(|u|^{p-2} u\right)\right\rangle\right| \\
\leq & \left.C \int_{\mathbb{R}^{3}}|\nabla| u\right|^{2} \|\left. u\right|^{p-4}|u||P-\bar{P}(t)| d x \\
\leq & \left.\left.\int_{\mathbb{R}^{3}} \frac{1}{32}|\nabla| u\right|^{2}\right|^{2}|u|^{p-4}+C|u|^{p+2}+|P-\bar{P}(t)|^{\frac{p+2}{2}} d x \\
\leq & C\left\{\|u\|_{L^{p}}^{\frac{2 p}{p-3}}\|u\|_{L^{p}}^{p}+\|\nabla d\|_{L^{p}}^{\frac{2 p}{p-3}}\|\nabla d\|_{L^{p}}^{p}\right\} \\
& +\frac{1}{32}\left(\left.\left.\int_{\mathbb{R}^{3}}|\nabla| u\right|^{2}\right|^{2}|u|^{p-4}+|u|^{p-2}|\nabla u|^{2} d x+\int_{\mathbb{R}^{3}}|\nabla d|^{p-2}\left|\nabla^{2} d\right|^{2} d x\right) .
\end{aligned}
$$

Similarly, applying (2.11) and by Sobolev's inequality and Cauchy's inequality, we get

$$
\begin{aligned}
& \left|\left\langle\triangle d \cdot \nabla d,|u|^{p-2} u\right\rangle\right|=\left|\left\langle\operatorname{div}\left(\nabla d \odot \nabla d-\frac{1}{2}|\nabla d|^{2} I\right),|u|^{p-2} u\right\rangle\right| \\
\leq & C\left\{\|u\|_{L^{p}}^{\frac{2 p}{p-3}}\|u\|_{L^{p}}^{p}+\|\nabla d\|_{L^{p}}^{\frac{2 p}{p-3}}\|\nabla d\|_{L^{p}}^{p}\right\} \\
& +\frac{1}{32}\left(\left.\left.\int_{\mathbb{R}^{3}}|\nabla| u\right|^{2}\right|^{2}|u|^{p-4}+|u|^{p-2}|\nabla u|^{2} d x+\int_{\mathbb{R}^{3}}|\nabla d|^{p-2}\left|\nabla^{2} d\right|^{2} d x\right) .
\end{aligned}
$$

Putting the above two inequalities into (2.10) we have

$$
\begin{aligned}
& \frac{d}{d t}\|u\|_{L^{p}}^{p}+\int_{\mathbb{R}^{3}}|u|^{p-2}|\nabla u|^{2} d x+\frac{1}{2} \int_{\mathbb{R}^{3}}|u|^{p-4}\left|\nabla\left(|u|^{2}\right)\right|^{2} d x \\
\leq \quad & C\left\{\|u\|_{L^{p}}^{\frac{2 p}{p-3}}\|u\|_{L^{p}}^{p}+\|\nabla d\|_{L^{p}}^{\frac{2 p}{p-3}}\|\nabla d\|_{L^{p}}^{p}\right\}+\frac{1}{32} \int_{\mathbb{R}^{3}}|\nabla d|^{p-2}\left|\nabla^{2} d\right|^{2} d x .
\end{aligned}
$$

Next differentiating $(1.1 \mathrm{~b})$ with respect to $x$, we have

$$
\partial_{x} d_{t}-\triangle \partial_{x} d=\partial_{x}\left(|\nabla d|^{2} d-u \cdot \nabla d\right)
$$


We multiply (2.13) by $|\nabla d|^{p-2} \partial_{x} d$ and integrate over $\mathbb{R}^{3}$ to obtain that

$$
\begin{aligned}
& \frac{1}{p} \frac{d}{d t}\|\nabla d\|_{L^{p}}^{p}+\int_{\mathbb{R}^{3}}|\nabla d|^{p-2}\left|\nabla^{2} d\right|^{2} d x+\frac{1}{4}(p-2) \int_{\mathbb{R}^{3}}|\nabla d|^{p-4}\left|\partial_{x}\left(|\nabla d|^{2}\right)\right|^{2} d x \\
= & \left\langle|\nabla d|^{p-2} \partial_{x} d, \partial_{x}\left(|\nabla d|^{2} d-u \cdot \nabla d\right)\right\rangle \\
= & \|\nabla d\|_{L^{p+2}}^{p+2}+\left\langle|\nabla d|^{p-2} \partial_{x} d, 2 \nabla d \nabla \partial_{x} d d\right\rangle+\left\langle\nabla \cdot\left(|\nabla d|^{p-2} \partial_{x} d\right), u \cdot \nabla d\right\rangle \\
\leq & C\left(\|\nabla d\|_{L^{p+2}}^{p+2}+\|u\|_{L^{p+2}}^{p+2}\right)+\frac{1}{64} \int_{\mathbb{R}^{3}}|\nabla d|^{p-2}\left|\nabla^{2} d\right|^{2} d x \\
\leq & C\left\{\|u\|_{L^{p}}^{\frac{2}{p-3}}\|u\|_{L^{p}}^{p}+\|\nabla d\|_{L^{p}}^{\frac{2}{p-3}}\|\nabla d\|_{L^{p}}^{p}\right\} \\
& +\frac{1}{32}\left(\int_{\mathbb{R}^{3}}|u|^{p-2}|\nabla u|^{2} d x+\int_{\mathbb{R}^{3}}|\nabla d|^{p-2}\left|\nabla^{2} d\right|^{2} d x\right) .
\end{aligned}
$$

Combining (2.12) and (2.14) gives

$$
\begin{aligned}
& \frac{d}{d t}\left(\|u\|_{L^{p}}^{p}+\|\nabla d\|_{L^{p}}^{p}\right)+\int_{\mathbb{R}^{3}}|u|^{p-2}|\nabla u|^{2}+|\nabla d|^{p-2}\left|\nabla^{2} d\right|^{2} d x \\
\leq & C\left\{\|u\|_{L^{p}}^{\frac{2 p}{p-3}}\|u\|_{L^{p}}^{p}+\|\nabla d\|_{L^{p}}^{\frac{p-3}{p-3}}\|\nabla d\|_{L^{p}}^{p}\right\} .
\end{aligned}
$$

Now by (1.5) we have $q=\frac{2 p}{p-3}$, thus $\|u\|_{L^{p}}^{\frac{2}{p-3}}$ and $\|\nabla d\|_{L^{p}}^{\frac{2}{p-3}}$ belong to $L^{1}[0, T]$. For $p$, we divide into two case:

Case 1. If $4 \leq p<+\infty$, then $\frac{p}{p-3} \leq p$. Thus combining (2.9) and (2.15) gives

$$
\begin{aligned}
& \frac{d}{d t}\left(\left\|\left(\nabla u, \nabla^{2} d\right)\right\|_{L^{2}}^{2}+\|(u, \nabla d)\|_{L^{p}}^{p}\right)+C\left\|\left(\nabla^{2} u, \nabla^{3} d\right)\right\|_{L^{2}}^{2} \\
\leq & C\left(\|u\|_{L^{p}}^{\frac{2 p}{p-3}}\|u\|_{L^{p}}^{p}+\|\nabla d\|_{L^{p}}^{\frac{2 p}{p-3}}\|\nabla d\|_{L^{p}}^{p}+1\right) .
\end{aligned}
$$

By Gronwall's inequality, we have

$$
\begin{aligned}
& \left\|\left(\nabla u, \nabla^{2} d\right)(t, \cdot)\right\|_{L^{2}}^{2}+\|(u, \nabla d)(t, \cdot)\|_{L^{p}}^{p}+C \int_{0}^{t}\left\|\left(\nabla^{2} u, \nabla^{3} d\right)(s, \cdot)\right\|_{L^{2}}^{2} d s \\
\leq & C \exp \left[C \int_{0}^{t}\left(\|u(s, \cdot)\|_{L^{p}}^{\frac{2 p}{p-3}}+\|\nabla d(s, \cdot)\|_{L^{p}}^{\frac{2 p}{p-3}}+1\right) d s\right]<+\infty,
\end{aligned}
$$

for all $t \in[0, T]$.

Case 2. If $3<p<4$. Multiplying (2.15) by $\left(\|u\|_{L^{p}}^{p}+\|\nabla d\|_{L^{p}}^{p}\right)^{\frac{3}{p-3}}$, we obtain

$$
\frac{d}{d t}\left(\|u\|_{L^{p}}^{p}+\|\nabla d\|_{L^{p}}^{p}\right)^{\frac{p}{p-3}} \leq C\left(\|u\|_{L^{p}}^{\frac{2 p}{p-3}}+\|\nabla d\|_{L^{p}}^{\frac{2 p}{p-3}}\right)\left(\|u\|_{L^{p}}^{p}+\|\nabla d\|_{L^{p}}^{p}\right)^{\frac{p}{p-3}}
$$

We add (2.17) to (2.9) to obtain

$$
\begin{aligned}
& \frac{d}{d t}\left[\left\|\left(\nabla u, \nabla^{2} d\right)\right\|_{L^{2}}^{2}+\left(\|u\|_{L^{p}}^{p}+\|\nabla d\|_{L^{p}}^{p}\right)^{\frac{p}{p-3}}\right]+C\left\|\left(\nabla^{2} u, \nabla^{3} d\right)\right\|_{L^{2}}^{2} \\
\leq & C\left(\|u\|_{L^{p}}^{\frac{2 p}{p-3}}+\|\nabla d\|_{L^{p}}^{\frac{2 p}{p-3}}\right)\left(\|u\|_{L^{p}}^{p}+\|\nabla d\|_{L^{p}}^{p}\right)^{\frac{p}{p-3}}
\end{aligned}
$$

By Gronwall's inequality, we get

$$
\begin{aligned}
& \left\|\left(\nabla u, \nabla^{2} d\right)(t, \cdot)\right\|_{L^{2}}^{2}+\left(\|u(t, \cdot)\|_{L^{p}}^{p}+\|\nabla d(t, \cdot)\|_{L^{p}}^{p}\right)^{\frac{p}{p-3}} \\
& +C \int_{0}^{t}\left\|\left(\nabla^{2} u, \nabla^{3} d\right)(s, \cdot)\right\|_{L^{2}}^{2} d s \leq C \exp \left[C \int_{0}^{t}\|(u, \nabla d)(s, \cdot)\|_{L^{p}}^{\frac{2 p}{p-3}} d s\right]<+\infty,
\end{aligned}
$$


for all $t \in[0, T]$.

Next for $|\alpha|=3$. For $I_{3,1}$, we need to use the following Moser-type inequality (see $[17$, p. 43]):

$$
\left\|D^{s}(f g)\right\|_{L^{2}} \leq C\left(\|g\|_{L^{\infty}}\left\|\nabla^{s} f\right\|_{L^{2}}+\|f\|_{L^{\infty}}\left\|\nabla^{s} g\right\|_{L^{2}}\right)
$$

Thus we have

$$
\begin{aligned}
\left|I_{3,1}\right| & =\left|\left\langle\partial_{x}^{2} \operatorname{div}(u \otimes u), \partial_{x}^{4} u\right\rangle\right| \\
& \leq C\left\|\nabla^{3}(u \otimes u)\right\|_{L^{2}}^{2}+\frac{1}{16}\left\|\nabla^{4} u\right\|_{L^{2}}^{2} \\
& \leq C\|u\|_{L^{\infty}}^{2}\left\|\nabla^{3} u\right\|_{L^{2}}^{2}+\frac{1}{16}\left\|\nabla^{4} u\right\|_{L^{2}}^{2} \\
& \leq C\left\{\|\nabla u\|_{L^{2}}^{\frac{5}{6}}\left\|\nabla^{4} u\right\|_{L^{2}}^{\frac{1}{6}}\|\nabla u\|_{L^{2}}^{\frac{1}{3}}\left\|\nabla^{4} u\right\|_{L^{2}}^{\frac{2}{3}}\right\}^{2}+\frac{1}{16}\left\|\nabla^{4} u\right\|_{L^{2}}^{2} \\
& \leq C\|\nabla u\|_{L^{2}}^{14}+\frac{1}{8}\left\|\nabla^{4} u\right\|_{L^{2}}^{2} .
\end{aligned}
$$

For $I_{3,2}$, we apply (2.11) and (2.19) to obtain that

$$
\begin{aligned}
\left|I_{3,2}\right| & =\left|\left\langle\partial_{x}^{2} \nabla \cdot\left(\nabla d \otimes \nabla d-\frac{1}{2}|\nabla d|^{2} I\right), \partial_{x}^{4} u\right\rangle\right| \\
& \leq C\left\|\nabla^{3}\left(\nabla d \otimes \nabla d-\frac{1}{2}|\nabla d|^{2} I\right)\right\|_{L^{2}}^{2}+\frac{1}{8}\left\|\nabla^{4} u\right\|_{L^{2}}^{2} \\
& \leq C\|\nabla d\|_{L^{\infty}}^{2}\left\|\nabla^{4} d\right\|_{L^{2}}^{2}+\frac{1}{8}\left\|\nabla^{4} u\right\|_{L^{2}}^{2} \\
& \leq C\left\{\left\|\nabla^{2} d\right\|_{L^{2}}^{\frac{5}{6}}\left\|\nabla^{5} d\right\|_{L^{2}}^{\frac{1}{6}}\left\|\nabla^{2} d\right\|_{L^{2}}^{\frac{1}{3}}\left\|\nabla^{5} d\right\|_{L^{2}}^{\frac{2}{3}}\right\}^{2}+\frac{1}{8}\left\|\nabla^{4} u\right\|_{L^{2}}^{2} \\
& \leq C\left\|\nabla^{2} d\right\|_{L^{2}}^{14}+\frac{1}{8}\left(\left\|\nabla^{4} u\right\|_{L^{2}}^{2}+\left\|\nabla^{5} d\right\|_{L^{2}}^{2}\right) .
\end{aligned}
$$

Similar in the proof of $(2.21), I_{3,3}, I_{3,4}$ can be bounded as follows:

$$
\begin{aligned}
\left|I_{3,3}\right|= & \left|\left\langle\partial_{x}^{3}(u \cdot \nabla d), \partial_{x}^{5} d\right\rangle\right| \\
\leq & C\left\|\partial_{x}^{3}(u \cdot \nabla d)\right\|_{L^{2}}^{2}+\frac{1}{16}\left\|\nabla^{5} d\right\|_{L^{2}}^{2} \\
\leq & C\left(\|\nabla d\|_{L^{\infty}}^{2}\left\|\nabla^{3} u\right\|_{L^{2}}^{2}+\|u\|_{L^{\infty}}^{2}\left\|\nabla^{4} d\right\|_{L^{2}}^{2}\right)+\frac{1}{16}\left\|\nabla^{5} d\right\|_{L^{2}}^{2} \\
\leq & C\left\{\left\|\nabla^{2} d\right\|_{L^{2}}^{\frac{5}{6}}\left\|\nabla^{5} d\right\|_{L^{2}}^{\frac{1}{6}}\|\nabla u\|_{L^{2}}^{\frac{1}{3}}\left\|\nabla^{4} u\right\|_{L^{2}}^{\frac{2}{3}}\right. \\
& \left.+\|\nabla u\|_{L^{2}}^{\frac{5}{6}}\left\|\nabla^{4} u\right\|_{L^{2}}^{\frac{1}{6}}\left\|\nabla^{2} d\right\|_{L^{2}}^{\frac{1}{3}}\left\|\nabla^{5} d\right\|_{L^{2}}^{\frac{2}{3}}\right\}^{2}+\frac{1}{16}\left\|\nabla^{5} d\right\|_{L^{2}}^{2} \\
\leq & C\left(\left\|\nabla^{2} d\right\|_{L^{2}}^{14}+\|\nabla u\|_{L^{2}}^{14}+\frac{1}{8}\left(\left\|\nabla^{4} u\right\|_{L^{2}}^{2}+\left\|\nabla^{5} d\right\|_{L^{2}}^{2}\right),\right.
\end{aligned}
$$

and

$$
\begin{aligned}
\left|I_{3,4}\right| & =\left|\left\langle\partial_{x}^{3}\left(|\nabla d|^{2} d\right), \partial_{x}^{5} d\right\rangle\right| \\
& \leq C\left\|\partial_{x}^{3}\left(|\nabla d|^{2} d\right)\right\|_{L^{2}}^{2}+\frac{1}{32}\left\|\nabla^{5} d\right\|_{L^{2}}^{2} \\
& \leq C\left(\left\|\nabla^{3}|\nabla d|^{2}\right\|_{L^{2}}^{2}+\left\||\nabla d|^{2}\right\|_{L^{\infty}}^{2}\left\|\nabla^{3} d\right\|_{L^{2}}^{2}\right)+\frac{1}{32}\left\|\nabla^{5} d\right\|_{L^{2}}^{2} \\
& \leq C\left(\|\nabla d\|_{L^{\infty}}^{2}\left\|\nabla^{4} d\right\|_{L^{2}}^{2}+\|\triangle d\|_{L^{\infty}}^{2}\left\|\nabla^{3} d\right\|_{L^{2}}^{2}+\frac{1}{32}\left\|\nabla^{5} d\right\|_{L^{2}}^{2}\right. \\
& \leq C\left(\left\|\nabla^{2} d\right\|_{L^{2}}^{14}+\left\{\left\|\nabla^{2} d\right\|_{L^{2}}^{\frac{1}{2}}\left\|\nabla^{5} d\right\|_{L^{2}}^{\frac{1}{2}}\left\|\nabla^{2} d\right\|_{L^{2}}^{\frac{2}{3}}\left\|\nabla^{5} d\right\|_{L^{2}}^{\frac{1}{3}}\right\}^{2}\right)+\frac{1}{16}\left\|\nabla^{5} d\right\|_{L^{2}}^{2} \\
& \leq C\left\|\nabla^{2} d\right\|_{L^{2}}^{14}+\frac{1}{8}\left\|\nabla^{5} d\right\|_{L^{2}}^{2} .
\end{aligned}
$$

Putting (2.20)-(2.23) into (2.4) for $|\alpha|=3$ and by (2.16) and (2.18), we arrive at

$$
\begin{aligned}
& \frac{d}{d t}\left(\left\|\nabla^{3} u\right\|_{L^{2}}^{2}+\left\|\nabla^{4} d\right\|_{L^{2}}^{2}\right)+\left\|\nabla^{4} u\right\|_{L^{2}}^{2}+\left\|\nabla^{5} d\right\|_{L^{2}}^{2} \\
& \leq C\left(\left\|\nabla^{2} d\right\|_{L^{2}}^{14}+\|\nabla u\|_{L^{2}}^{14}\right) \leq+\infty
\end{aligned}
$$

Integrating the above inequality with respect to time from 0 to $t \in[0, T]$ and by $(2.3)$, we conclude $u(T, \cdot) \in H^{3}\left(\mathbb{R}^{3}\right)$ and $d(T, \cdot)-a \in H^{4}\left(\mathbb{R}^{3}\right)$. Thus the proof of Theorem 
1.1 is completed.

\section{Acknowledge}

The authors' research was supported Supported by National Natural Science Foundation of China-NSAF (Grant No. 10976026) and by National Natural Science Foundation of China-NSAF (Grant No. 11271305).

\section{References}

[1] J. T. Beale, T. Kato, A. Majda, Remarks on the breakdown of smooth solutions for the 3-D Euler equation. Commun. Math. Phys. 94: 61-66.

[2] P. G. DE Gennes, The Physics of Liquid Crystals. Oxford, 1974.

[3] J. L. Ericksen, Hydrostatic theory of liquid crystal. Arch. Ration. Mech. Anal. 9 (1962), 371-378.

[4] L. Escauriaza, G. Seregin, V. Sverák, $L_{3, \infty}$ solutions of the Navier-Stokes equations and backward uniqueness. Russ. Math. Surv. 58 (2003), 211-250.

[5] M. C. Hong, Global existence of solutions of the simplified Ericksen-Leslie system in $\mathbb{R}^{2}$. Calc. Var. Partial Differential Equations 40 (2011), 15-36.

[6] M. C. Hong, Z. P. Xin, Global existence of solutions of the Liquid Crystal flow for the OseenFrank model in $\mathbb{R}^{2}$. Adv. Math., 231 (2012), 1364-1400.

[7] T. Huang, C. Y. Wang, Blow up criterion for nematic liquid crystal flows. Comm. Partial Differ. Equ. 37 (2012), 875-884.

[8] O. Ladyzhenskaya, Uniqueness and smoothness of generalized solutions of Navier-Stokes equations. Zap. Nauv cn. Sem. Leningrad. Otdel. Mat. Inst. Steklov. (LOMI) 5 (1967), 169-185.

[9] J. Leray, Sur le mouvement d'un liquide visqueux emplissant l'espace. Acta Math. 63 (1934) 193-248.

[10] F. M. Leslie, Some constitutive equations for liquid crystals. Arch. Ration. Mech. Anal. 28 (1962), 265-283.

[11] F. H. Lin, Nonlinear theory of defects in nematic liquid crystals; phase transition and flow phenomena. Commun. Pure Appl. Math. 42 (1989), 789-814.

[12] F. H. Lin, C. Liu, Nonparabolic dissipative systems modeling the flow of liquid crystals. Commun. Pure Appl. Math. 48 (1995), 501-537.

[13] F. H. Lin, C. Liu, Partial regularity of the dynamic system modeling the flow of liquid crystals. Discrete Contin. Dyn. Syst. 2 (1996), 1-22.

[14] F. H. Lin, J. Lin, C. Y. Wang, Liquid crystal flows in two dimensions. Arch. Rational Mech. Anal. 197 (2010), 297-336.

[15] F. H. Lin, J. Lin, C. Y. Wang, On the uniqueness of heat flow of harmonic maps and hydrodynamic flow of nematic liquid crystals. Chinese Ann. Math. 31B: 921-928.

[16] Q. Liu, J. Zhao, Logarithmical Blow-up Criteria for the Nematic Liquid Crystal Flows, printed.

[17] A. Majda, Compressible fluid flow and system of conservation laws in several space variables. Applied Mathematical Sciences, 53, Springer- Verlag, NewYork, 1984.

[18] G. Prodi, Un teorema di unicit per le equationi di Navier-Stokes. Ann. Mat. Pura Appl. (4) 48 (1959), 173-182.

[19] J. Serrin, The initial value problem for the Navier-Stokes equations. Nonlinear Problems (Proc. Sympos., Madison, Wis.). University of Wisconsis Press, Madison, 69-98, 1963.

[20] X. Xu and Z. Zhang, Global regularity and uniqueness of weak solution for the 2-D liquid crystal flows. J. Differential Equations 252 (2012), 1169-1181. 